\title{
Determining the Vulnerabilities of the Power Transmission System
}

\author{
B. A. Carreras \\ D. E. Newman \\ I. Dobson \\ Depart. Fisica \\ Physics Dept. \\ ECpE Department \\ Universidad Carlos III University of Alaska \\ Madrid, Spain Fairbanks AK 99775 \\ Iowa State University \\ bacarreras@gmail.com denewman@alaska.edu dobson@iastate.edu
}

\begin{abstract}
Determining the vulnerabilities in power transmission systems requires two distinct steps because most large blackouts have two distinct parts, the triggers/initiating event followed by the cascading failure. Finding the important triggers for large blackouts is the first and standard step. Next, the cascading part of the extreme event (which can be long or short) is critically dependent on the "state" of the system; how heavily the lines are loaded, how much generation margin exists, and where the generation exists relative to the load. However, during large cascading events there are some lines whose probability of overloading is higher that the others. Statistical studies of blackouts using the OPA code allow the identification of such lines or groups of line for a given network model, thereby providing a technique for identifying at risk (or critical) clusters. This paper addresses both parts of the vulnerability question.
\end{abstract}

\section{Introduction}

In designing power transmission systems, the standard practice is the application of the n- 1 criterion to most lines and some higher order criteria for a few lines. This has been a rather effective way to establish robust power transmission systems. However, there are several problems that intrinsically limit the overall effectiveness of such approach.

One of the problems with this approach is the impossibility of applying higher order criteria, n-2, n-3 and so on, to all components of the system. The number of potential combinations increases so rapidly that it makes the calculations impossible. This is important because to prevent large cascading events requires the testing of multiple simultaneous failures, a rare but not impossible scenario.

A second problem is that all of these tests should be done under all possible conditions of the power system if they are to be effective in evaluating risk. An initiating event for an extreme event is the combination of both a triggering event and the state of the system. In this type of analysis of the robustness of the grid, the goal should be the identification of initiating events. To test all possibilities is again impossible.

Therefore, it is important to complement the standard test of power systems with other ways of detecting vulnerabilities of the system to combinations of initiating events and the propagation of the cascading failures.

Here, two complementary approaches to the standard one are discussed. They are applied to the study of vulnerabilities in the western interconnect using test networks. The first approach is based on the use of a dynamical model, the OPA (ORNL-PSercAlaska) model [1-3], which allows exploration of multiple states of the power system and carries out vulnerability tests under varying conditions. Each network system is tested at many different times for failures. These failures may be chosen randomly or targeting specific components of the networks. The method is a Monte-Carlo like approach to the vulnerability issue, but with the background system evolving in a self-consistent manner thereby sampling many system states.

The second approach consists of examining high frequency simultaneous overloading and failures of lines using a synchronization matrix [4]. This is done during the dynamical evolution of the system using the OPA model. This allows for the determination of critical clusters of lines in the system that are important in the propagation of the cascading failures.

It is important to note that lines that are critical in triggering large blackouts are not necessarily the same lines that are critical in the propagation of the cascade. In the calculations that follow, it is seen that these two sets of lines are in general actually disjoint. This 
becomes an important issue when dealing with mitigation since strengthening the triggering elements will not necessarily strengthen the critical elements in the cascading failure group.

This paper is focused mostly on the methods. We discuss the methods and their results but intentionally do not identify the particular lines that have been found to be more likely to cause large damage to the system or foster the propagation of the cascades. This information can be obtained by applying the methods, but is not documented in the paper. Section 2 introduces the WECC 225 and 1553 bus models we use for the work. Section 3 discusses the identification of the most important elements (or sets of elements) for triggering the initiating events and section 4 investigates the identification of the critical clusters that are important in the propagation phase of the cascading failure. Finally, section 5 is a brief summary of the results.

\section{Validation of OPA for the WECC networks}

The OPA model for the dynamics of series of blackouts in power transmission systems $[1,2,3]$ has shown how the slow opposing forces of load growth and network upgrades in response to blackouts could self organize the power system to dynamic equilibrium. Blackouts are modeled by overloads and outages of lines determined in the context of LP dispatch of a DC load flow model. This model has been found to show complex dynamical behavior $[1,2]$ consistent with that found in the NERC historical data for blackouts [5].

In the work presented here, the focus is on the Western Interconnect as represented by two reduced models. One is a very reduced 225 node model which allows relatively fast exploration of methods and measures, while the other is a 1553 node model which has much more fidelity to the static and dynamic characteristics of the real network, though it is still significantly reduced. The 1553 node model has 2114 lines. Because the OPA model simulates long series of blackouts as the power grid slowly upgrades, it is very time consuming and therefore difficult to gather a large statistical base.

In order to validate OPA and the network models, system parameters characteristic of the WECC (or in some cases the California region) are used. These include a demand growth rate of $2 \%$ per year (between the $1.93 \% / \mathrm{yr}$ for California and the $2.3 \% / \mathrm{yr}$ for the WECC), and an average generation capacity margin of $20 \%$.

The 6 main parameters that are inputs for OPA are shown in table 1 . The daily increase rate, $\lambda$, the generation capacity margin, $\Delta \mathrm{P} / \mathrm{P}$, the random probability of failure of a component adjusted for system size, $\mathrm{p}_{0}$, and the load variance parameter, $\gamma$, are all set within a range by the real network characteristics. This leaves only 2 parameters to be chosen to capture the system dynamics.

\begin{tabular}{|c|c|}
\hline Parameter & Description \\
\hline$\lambda$ & $\begin{array}{c}\text { Daily rate of increase of the } \\
\text { demand }\end{array}$ \\
\hline $\mathrm{p}_{0}$ & $\begin{array}{c}\text { Probability of failure of a } \\
\text { component by a daily random } \\
\text { event }\end{array}$ \\
\hline $\mathrm{p}_{1}$ & $\begin{array}{c}\text { Probability of an overloaded } \\
\text { line outaging }\end{array}$ \\
\hline$\mu$ & $\begin{array}{c}\text { Rate of upgrade of the } \\
\text { overloaded lines after a } \\
\text { blackout }\end{array}$ \\
\hline$\Delta \mathrm{P} / \mathrm{P}$ & Capacity margin \\
\hline$\gamma$ & $\begin{array}{c}\text { Controls the variance of the } \\
\text { loads }\end{array}$ \\
\hline
\end{tabular}

Table 1 The main OPA input parameters

The first of these parameters is simply the frequency of blackouts. In OPA studies, a blackout is an event whose size, $\mathrm{S}=$ Load shed/Power demand, is greater than 0.00001 . This definition is not the same as the definition of a reportable blackout from the NERC point of view.

The NERC data arise from government incident reporting requirements. The thresholds for the reporting of an incident include uncontrolled loss of $300 \mathrm{MW}$ or more of system load for more than $15 \mathrm{~min}$ from a single incident, load shedding of $100 \mathrm{MW}$ or more implemented under emergency operational policy, loss of electric service to more than 50000 customers for $1 \mathrm{~h}$ or more, and other criteria detailed in U.S. Department of Energy form EIA-417.

The NERC definition is rather complex, so in the present calculations, an effective criterion used is the loss of $300 \mathrm{MW}$ or more. Therefore, a blackout is an event with $\mathrm{S}>0.003$. With this choice of criterion and for the parameters of Table 2, the frequency of the blackouts is between 0.03 and 0.04 , depending on the value of $\mathrm{p} 1$. This is consistent with the value of the blackout frequency for the western interconnect of 0.37 between 1984 and 2006. 


\begin{tabular}{|c|c|c|}
\hline $\begin{array}{c}\text { Variable } \\
\text { Symbol }\end{array}$ & $\begin{array}{c}\text { Value - 225 } \\
\text { bus }\end{array}$ & $\begin{array}{c}\text { Value - 1553 } \\
\text { bus }\end{array}$ \\
\hline$\mu$ & 1.07 & 1.07 \\
\hline$p_{0}$ & $5.0000 \mathrm{e}-05$ & 0.0001 \\
\hline$p_{1}$ & 0.15 & $0.10-0.05$ \\
\hline$\gamma$ & 1.37 & 1.15 \\
\hline$\Delta \mathrm{P} / \mathrm{P}$ & 0.2 & 0.2 \\
\hline$\lambda$ & 1.00005 & 1.00005 \\
\hline
\end{tabular}

Table 2 OPA parameters for the 225 and 1553 node systems

Next we look at the probability distribution of sizes of the blackouts. This is a critically important measure for many complex systems as one of the characteristics of these systems which make risk estimation difficult is the non-normal distribution often found. The power transmission system has been found to have a power law distribution making the largest events dominate the risk which motivates the investigation of determining the systems vulnerability to those failures [6].

Figure 1 shows 1- CDF (cumulative distribution function) as calculated using the rank function for the OPA calculations for 225 node model and the real WECC data. The results are plotted on a log-log graph in order to highlight the power law nature (a straight line on the log-log plots) of the data. Reasonable agreement is found over much of the range however, due to the limited system size, the power law tail for the OPA results extends less than a decade and rolls off sooner then the real data.

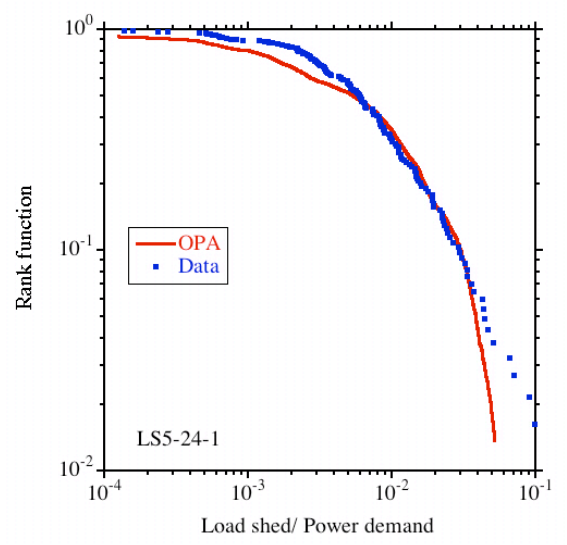

Fig. 1. 1-CDF of normalized load shed in the WECC 225 node network

In figure 2 the same comparison is done for the OPA calculation using the 1553 node model and the real WECC data. In this case even better agreement is found, with the power law extending slightly over a decade well into the tail. This improved agreement demonstrates the importance of using adequate system sizes for some of the measures.

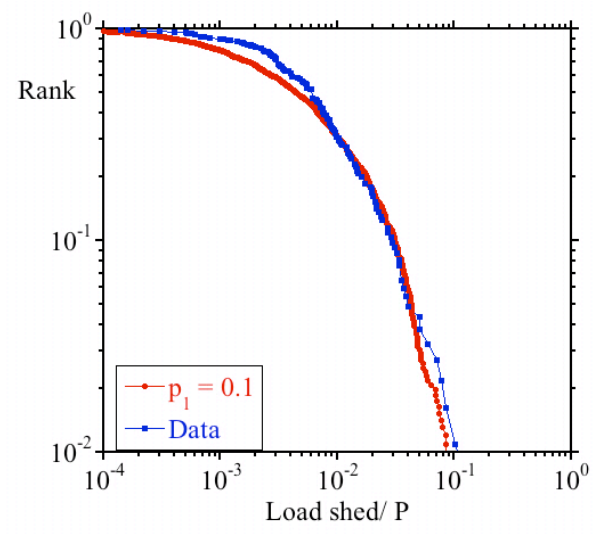

Fig. 2. 1-CDF of normalized load shed in the WECC 1553 node network

There is public data for about a decade of forced transmission line outages in the Pacific Northwest [7]. These data have been grouped into cascades to obtain a probability distribution of the number of line outages in each cascade [8]. We compare the distribution of line outages from the 1553 node OPA simulations for two values of the $\mathrm{p} 1$ parameter with the observed data. These results are shown in figure 3 . While both values give remarkably good results, $\mathrm{p} 1=0.1$ gives slightly better in the last few points.

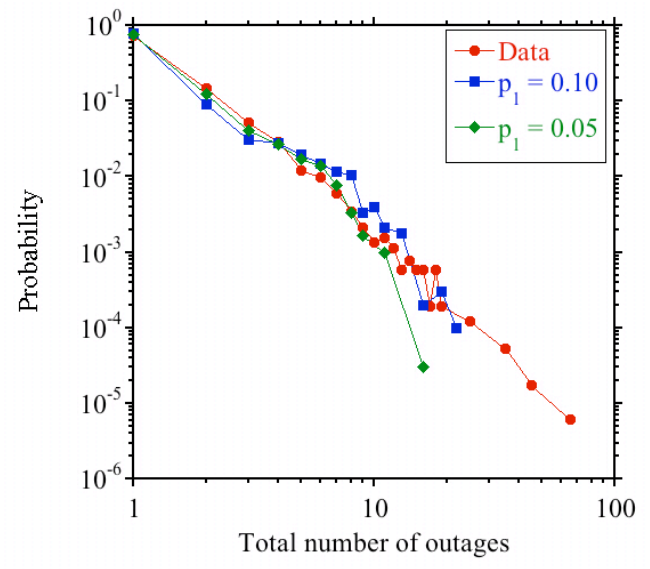

Fig. 3. The distribution of total line outages from the OPA calculation for the WECC 1553 node network and parameters of TABLE 2 compared with the data 
Using both the WECC 225 node and 1553 node networks a very reasonable agreement has been obtained between the statistical data on blackouts from the Western interconnect and the model calculations from OPA, validating the models for these characteristics.

A set of parameters has been found giving sufficient agreement with the data to allow the determination of the case as a reference case for the WECC 1553 network.

Using these models and parameters, it is now possible to investigate the most vulnerable lines leading to and during a cascading event and the regions more likely to blackout. It is also possible to identify the dominant blackout patterns and the sectors of the grid that they affect.

\section{Vulnerability of the WECC networks to a limited number of line outages}

A few simultaneous line outages are common incidents that can be caused by weather or similar events. They then may be the trigger of an extreme event. We examine the vulnerability of a network model to this type of failure under varying conditions of the network and try to determine which lines can cause most damage to the system.

The approach followed in these studies is:

1. Carry out a time evolution calculation of the network using the OPA code over a long period of time. Periodically during the evolution, an outage of a given number $\mathrm{k}$ of lines chosen randomly is triggered.

2. From these calculations, a list of lines that cause the worst and most probable damage to the system is produced. From the list, several strategies are applied in order to identify subsets of lines that cause the most damaging events.

3. The time evolution calculations are done again but now specifically targeting the lines selected by the strategies applied before. This allows the process to focus on the outages that causes maximum damage to the system.

Based on these calculations, it is possible to identify some of the worst possible initiating events that can lead to extreme events in the network under study.

There are many possible strategies to follow in selecting the lines to target based on different measures of "badness" for an outage. The following two strategies are the ones applied here:

Strategy I - Each line selected randomly is associated with a measure of it's impact: the size of the blackout divided by the number of lines that outage simultaneously. If a line appears more than once in the list, the measures are added. Then lines are ordered by the size of the measure. Only measures greater than 0.05 are considered.

Strategy II - Select the lines that outage more often during the normal (unperturbed) runs. Then lines are ordered by how often they outage.

Once lines are selected, they are combined to maximize their impact on the system.

In applying this method to the WECC 225 node network, calculations are done over a period of 105 days and line outages are triggered every 500 days. The choice of 500 days is made in order to avoid having the dynamical evolution of the system changed by the externally triggered failures, which would cause an artificial system state.

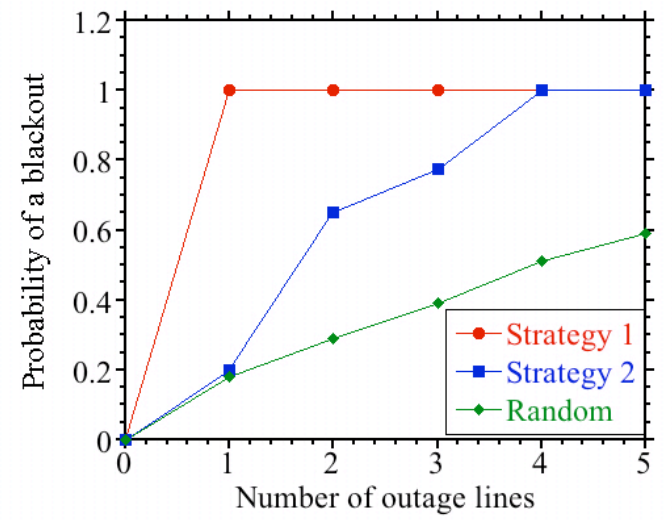

Fig. 4. Probability of a blackout by the outage of $k$ lines in the WECC 225 node network

For the WECC 225 node network, using the correct strategy, large blackouts can be triggered by the outage of only 2 or 3 lines. This is shown in Fig. 4. This figure shows the probability of a blackout by the outage of $k$ lines, with $\mathrm{k}$ varying from 1 to 5 . We can see that the strategies we have used are more effective in causing a blackout than the random choice of the lines. However, many of the blackouts triggered this way are not significant. Therefore, it is better to look for other measures to better assess the efficiency of this approach.

Fig. 5 shows the probability of a large blackout by the outage of $\mathrm{k}$ lines following the same strategies as in Fig. 4. Here, S, equal to the total load shed divided by power demand, is used as a measure of a blackout size. A large blackout is defined as one that causes a load shed of $10 \%$ or more of the total power demand.

As one might expect, by choosing the lines randomly there is very low probability of causing large blackouts. However, once the information from 
random failures is collected, strategies can be developed that lead to the triggering of large blackouts with relatively high probability. In this way, critical lines are identified.

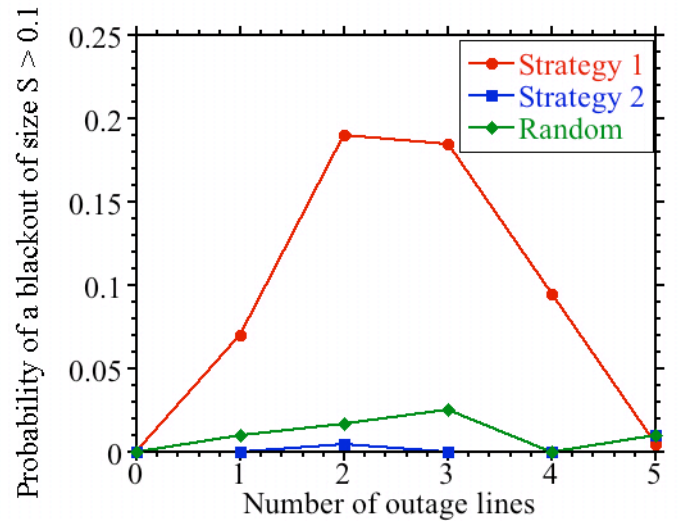

Fig. 5. Probability of a large blackout by the outage of $k$ lines in the WECC 225 node network

In developing a strategy, first a list of the most likely lines to cause a large blackout is compiled. Then a choice is made of the most likely combination of lines that maximize damage to the network. In Fig. 6 the probability of producing a large blackout using 10 different groups of three lines that have been chosen on the basis of the damage they cause when selected randomly is shown. As it is clear from the figure, there are groups of lines that are very much more effective than others in triggering large blackouts.

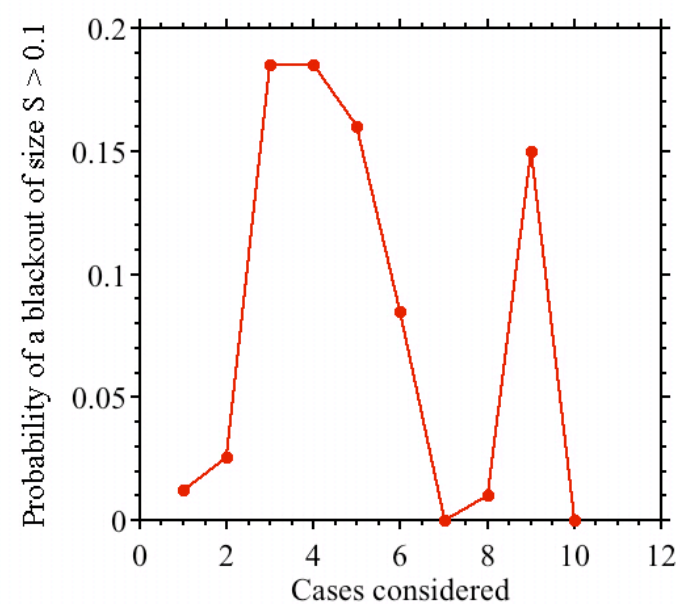

Fig. 6. Probability of producing a large blackout by 10 different groups of three lines in the WECC 225 node network

Fig. 7 shows the average size of the blackout caused by the same selection of triggering lines as the ones in Fig. 5. The error bars in Fig. 7 represent the standard deviation. Again, the average size of the blackouts caused by random selection of the triggers is smaller than the one caused by targeted lines.

The results plotted in Fig. 4 give some idea of the potential damage caused by the line outages; however, they do not provide a proper measure of that damage. The reason is that the distribution of possible outcomes of a simultaneous failure of a few lines is far from being a Gaussian distribution. Therefore, the mean value and standard deviation do not provide adequate information.

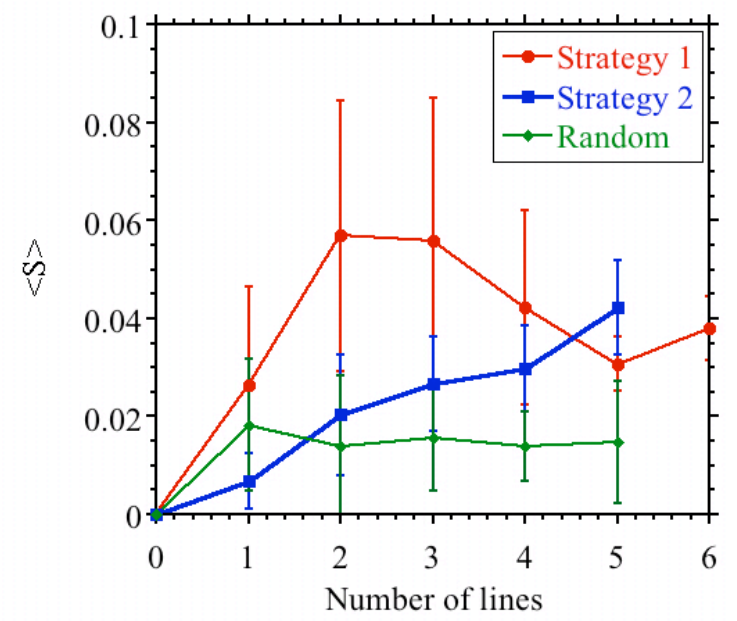

Fig. 7. Average size of the blackouts caused by the same selection of triggering lines as the ones in Fig. 2 in the WECC 225 node network. The error bars represent the standard deviation.

For instance, Fig. 8 shows the PDF of the blackout size for all cases triggered by the simultaneous outages of three targeted lines following strategy 1. This distribution has a slowly falling off tail, and clearly the mean and the standard deviation given in Fig. 7 do not reflect this structure. This PDF indicates that it is possible to cause very large events, up to $20 \%$ of the total power. Although the triggering of these extreme events is always possible, their occurrence depends on the timing of the outages; that is, what the system state is when the trigger occurs. That is why there are such different possible outcomes from the same type of trigger event. Once again, it is clearly shown the importance of examining the vulnerability of the system under varying conditions of the system.

From Fig. 8, the probability of an event in the range $15 \%$ to $20 \%$ of power lost is about 0.05 . However, that is the probability of such a failure when the three chosen lines fail simultaneously. Therefore, the probability of such event would be 0.05 times the probability of these three lines failing simultaneously 
and that is a very low probability. But if it is the risk of extreme events that must be reduced, it is the occurrence of unlikely but possible scenarios that one must prevent.

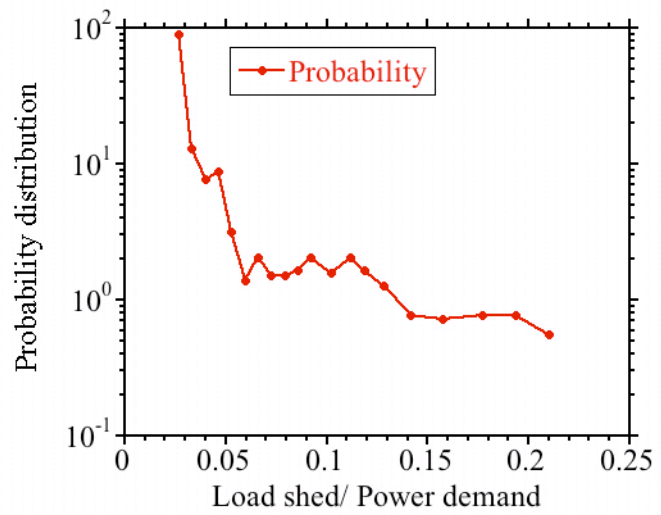

Fig. 8. PDF of the blackout size for all cases triggered by the simultaneous outages of three targeted lines following strategy 1 in the WECC 225 node network.

In going from the WECC 225 node network to the WECC 1553 node network the difficulty of the problem increases considerably because the calculations take considerably longer and the number of possible line failures also dramatically increases. In the case of the WECC 1553 node network, the same level of statistical samples that was possible for the smaller network, cannot be accumulated. However, the evaluation of the vulnerability of this system is still feasible.

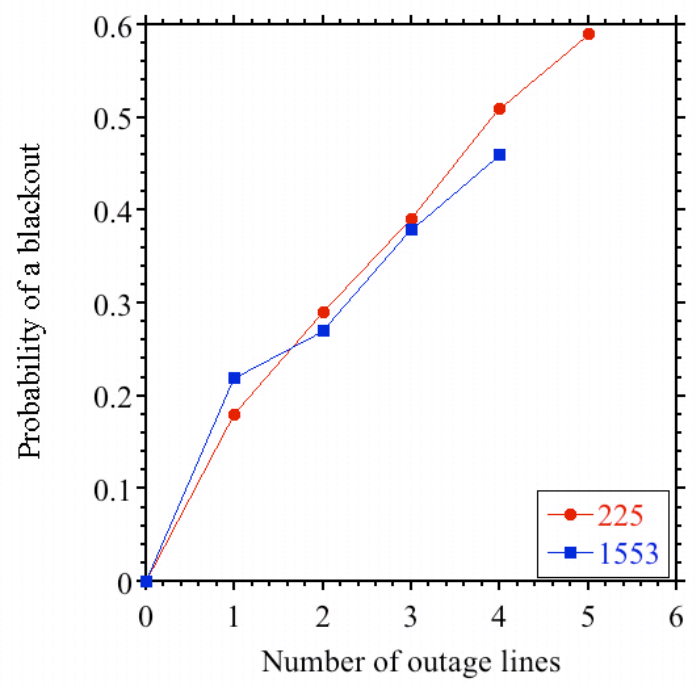

Fig. 9. Probability of triggering a blackout by the outage of $k$ lines chosen randomly for the two WECC networks under study.
It is interesting to observe that for random choice of lines, the probability of a blackout is practically the same for both networks. This is shown in Fig. 9, where the probability of triggering a blackout by the outage of $\mathrm{k}$ lines chosen randomly is plotted for the two WECC networks.

For the WECC 1553 node network, a single strategy is applied. This strategy is based on the most successful one for the smaller network, strategy 1 . Following this strategy the results for the probability of a blackout when $\mathrm{k}$, $\mathrm{k}$ varying from 1 to 4 , shown in figure 10, lines are outaged are similar to the results for strategy 1 in the WECC 225 node network (Fig. 4). This similarity of results when changing the network model size allows some confidence in the validity of extrapolating this work to the real network.

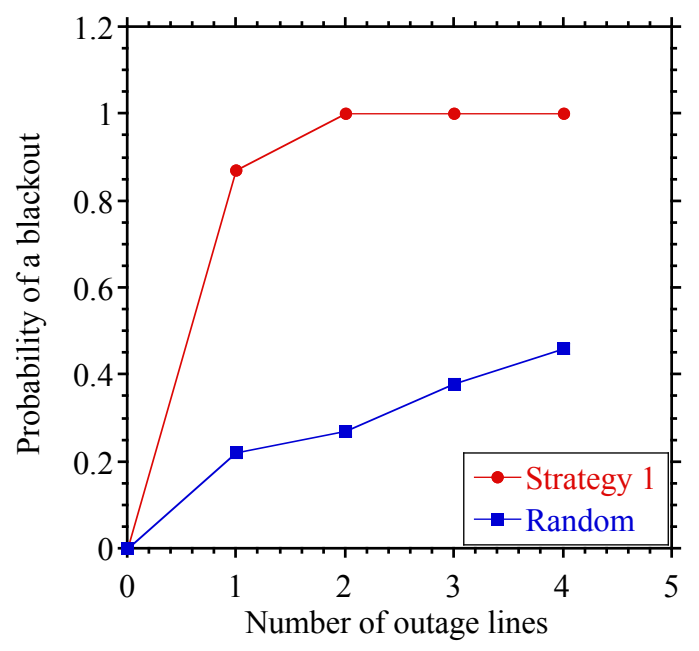

Fig. 10. Probability of a blackout by the outage of $k$ lines in the WECC 1553 node network

Following strategy 1 , the probability for triggering large blackouts in the WECC 1553 node network is similar to the results obtained for the WECC 225 node network. Fig 11 for the WECC 1553 node network corresponds to Fig 5 for the WECC 225 node network. Again the probability of a large blackout is considerably higher for targeted lines than for randomly chosen lines. With strategy 1 it is possible to reach probabilities of the order of $10 \%$ to $30 \%$. As before, the result depends on the state of the network.

As the size of a network increases and in normal operation, the relative size of the large blackouts decreases. So, in normal operation, the frequency of a blackout greater than $10 \%$ of the system size is 0.011 for the WECC 225 node network and it is less than 0.0003 for the WECC 1553 node network. However, the probability of triggering large blackouts with 
targeted lines is of the same order for both networks, as can be seen by comparing Figs. 5 and 10 .

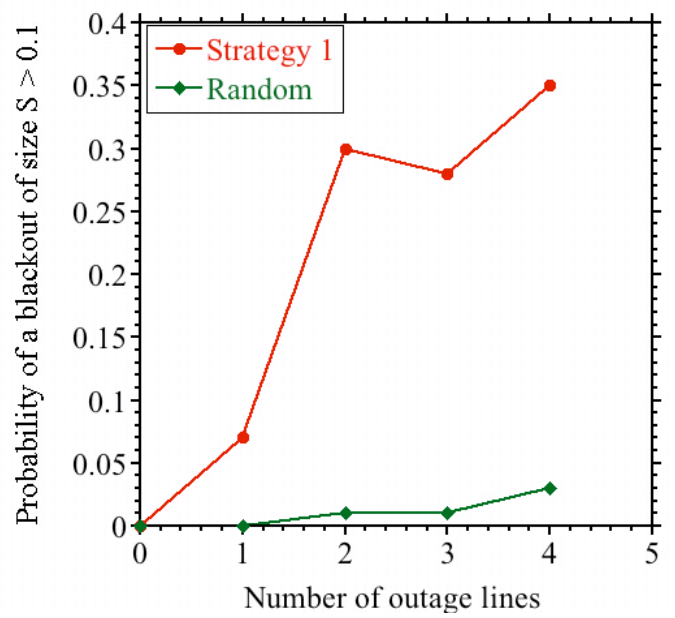

Fig. 11. Probability of a large blackout by the outage of $k$ lines in the WECC 1553 node network.

In addition, the size of the triggered blackouts seems to be similar for the two networks. Fig. 12 shows a plot similar to Fig. 7 for the WECC 1553 node network. In this plot, the average size of the triggered blackout is given as a function of the number of line outages triggering it. Error bars give the standard deviation.

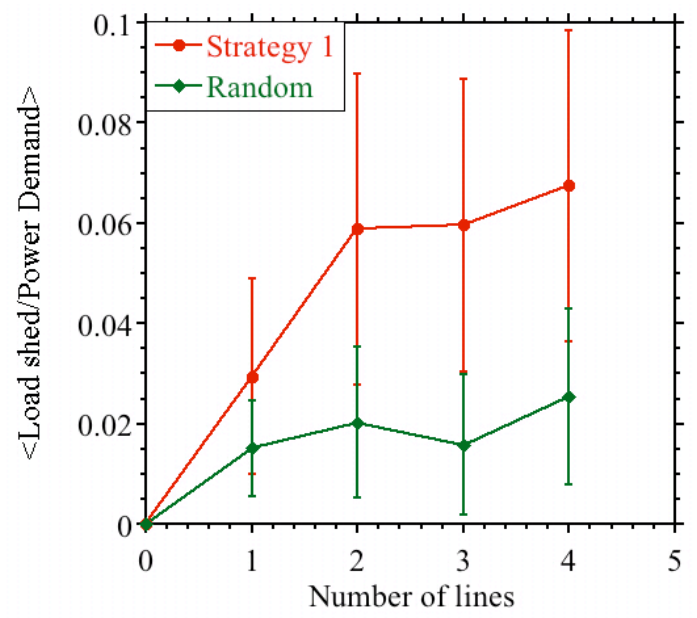

Fig. 12. Average size of the blackouts caused by the same selection of triggering lines as the ones in Fig. 8 in the WECC 1553 node network. The error bars represent the standard deviation.

In summary, following this approach it is possible to select a group of lines for which an outage can trigger large blackouts in the network. Having these lines, it is then possible to design mitigation methods that can reduce the likelihood of such events. These critical trigger lines have been identified for both WECC network models tested.

\section{Critical line clusters and cascade propagation}

During large blackouts there are some lines whose probability of overloading, and therefore participating in the cascade, is higher that the others. Statistical studies of blackouts using the OPA code allow the identification of such lines for a given network model, thereby providing a technique for identifying at risk (or critical) lines or groups of lines. These lines play a critical role in the propagation of large events because they are likely to fail during the propagation of the cascade. Therefore, it is important to identify them.

To identify these critical lines, first a record is kept of lines that overload simultaneously during a large blackout. Here, these groups of lines are called critical clusters of lines.

Then, a synchronization approach [4] is used for the identification. This approach has been successfully applied in the study of chaotic systems and it has already been applied to study coupled infrastructure systems [9].

For a given model network, the following matrix is constructed,

$$
S(i, j)=\sum_{\text {large blackouts }} \mathrm{Ov}(\mathrm{i}) \mathrm{Ov}(\mathrm{j})
$$

Here, Ov(i) is a variable that takes only two values, 1 if the line $i$ overloads during the blackout and 0 if it does not. In Eq. (1) and for each pair of lines, i and j, the sum is taken over all large blackouts. The definition of large blackout is flexible and depends on the specifics of interest to be studied. For the moment and following the previous section, a large blackout is defined as a blackout with over $10 \%$ load shed .

Note that $\mathrm{S}(\mathrm{i}, \mathrm{i})$ is equal to the total number of large blackouts in which line $\mathrm{i}$ has overloaded. $\mathrm{S}(\mathrm{i}, \mathrm{j})$ is equal to the total number of large blackouts in which lines $\mathrm{i}$ and $\mathrm{j}$ have both overloaded. Therefore, this matrix has the combined information of the frequency of overloading of the two lines as well as their synchronization or correlation

Once the synchronization matrix is constructed and in order to visualize its structure, the elements of the matrix corresponding to frequencies greater than a given one, S0, are plotted. An example is shown in Fig. 13. In this figure and for the WECC 225 node network, the elements of the synchronization matrix for lines 
that overload together with a frequency greater than $\mathrm{S} 0=4$ times in 105 are plotted. A clear diagonal line is seen, which tells the obvious, that each line is strongly synchronized with itself. But more interestingly there are a few clusters. They are groups of critical lines to be identified. These are the critical clusters of lines.

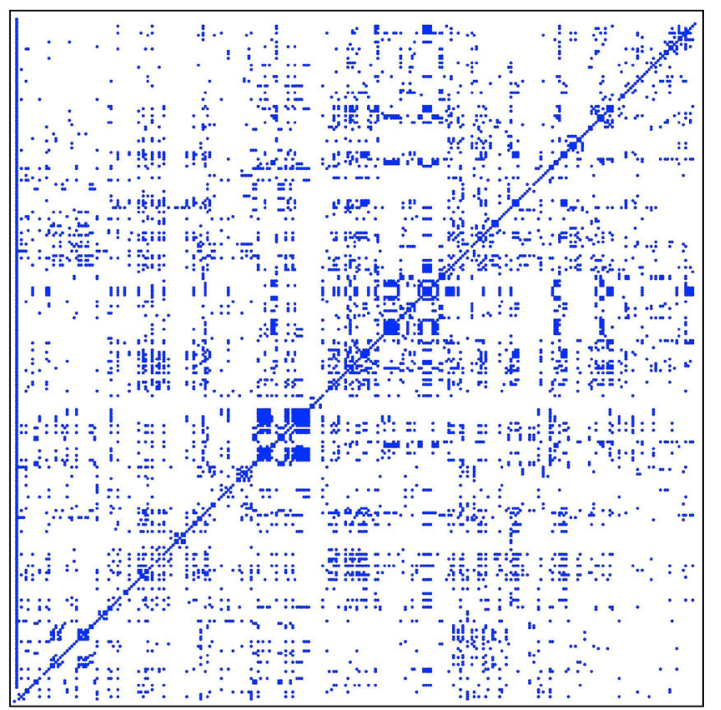

Fig. 13. Elements of the synchronization matrix for lines that overload together with a frequency greater than $S 0=4$ times in 105 for the WECC 225 node network

Although this visualization is a very useful way of giving a sense of the number and relative importance of the critical line clusters, it is hard to do a detailed diagnostic of those clusters. This is even more difficult for larger network models. For instance, for the WECC 1553 network the matrix is a $2114 \times 2114$ matrix. However, by changing the value of $\mathrm{S} 0$, it is possible to get a feeling for the importance of the clusters. Figs. 14 to 17 show the synchronization matrices for the WECC 1553 node network and for four values of S0, 1,3, 4 , and 6 in $3 \times 104$ days. As the threshold frequency is increased, the most critical of the clusters of lines emerge.

Although the matrix in Fig. 17 looks very sparse, it still involves 447 lines which have frequencies of overloading in large blackouts above 6 times in 3x104 days. For this case, the cluster with the largest frequency involves 20 lines and is a disjoint cluster. The lines are located in the 8 separate clusters of nodes that are drawn in Fig. 18. The critical lines are the ones numbered in the figure. The disjoint nature of the critical cluster is not surprising. Because the cascade propagation in a power system is non-local so are the clusters.

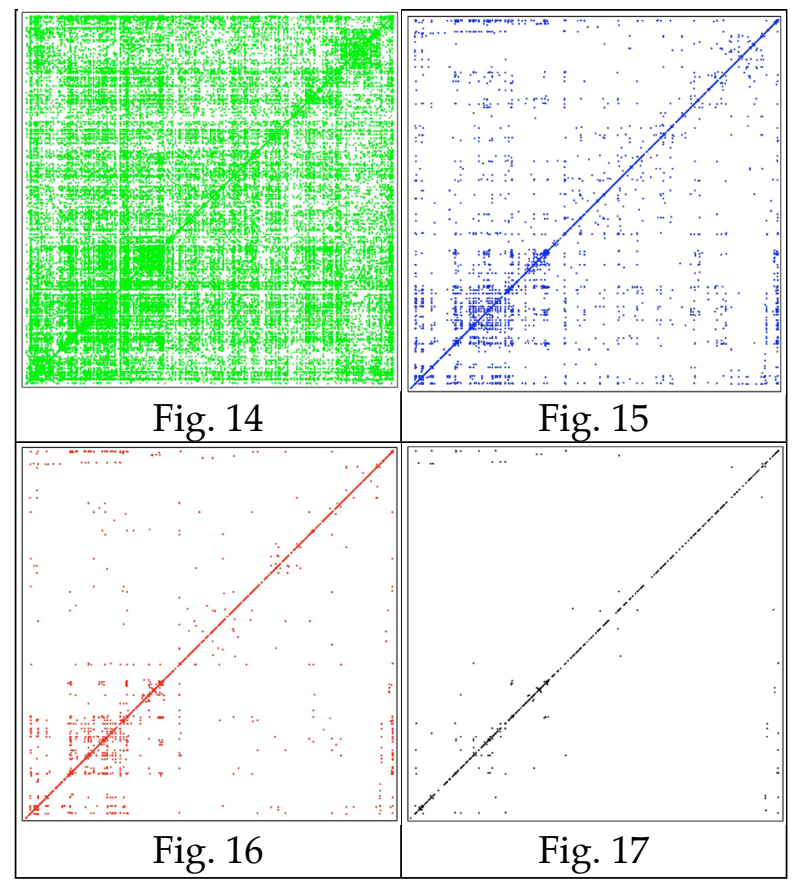

Figs. 14 to 17. Synchronization matrices for the WECC 1553 node network for four values

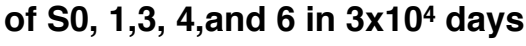
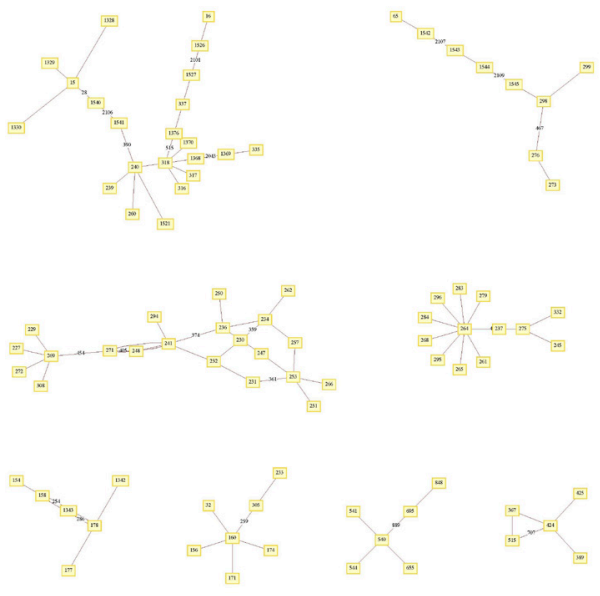

Fig. 18. Cluster of nodes where the 20 lines constituting the highest frequency critical cluster for the WECC 1553 node network are located. Critical lines are numbered (all numbering is artificial to prevent any correspondence with real data).

Now, post processing of the data is necessary to extract the quantitative information. To extract the 
quantitative information from the synchronization matrices, we use a system of equivalence classes. The synchronization between lines can be used to establish an equivalence relation between lines. Then the clusters are equivalence classes and the matrices can be analyzed from this perspective.

For instance, two lines $\mathrm{i}$ and $\mathrm{j}$ will be made equivalent if $\mathrm{S}(\mathrm{i}, \mathrm{j})>\mathrm{S} 0$. Here, $\mathrm{S} 0$ is a predetermined value. In this way, the clusters for Figs. 14 to 17 are identified and a list of lines for each case can be made. From these lists, Fig. 18 was constructed.

The list of lines obtained this way is different from the list produced in the previous section based on triggering large blackouts. Each of these sets of lines plays a different role towards the vulnerability of the network and both need to be identified. Fig. 19 is a plot showing the two sets of lines, in red are the identified trigger lines and in blue the critical lines from the cluster in Fig. 18.

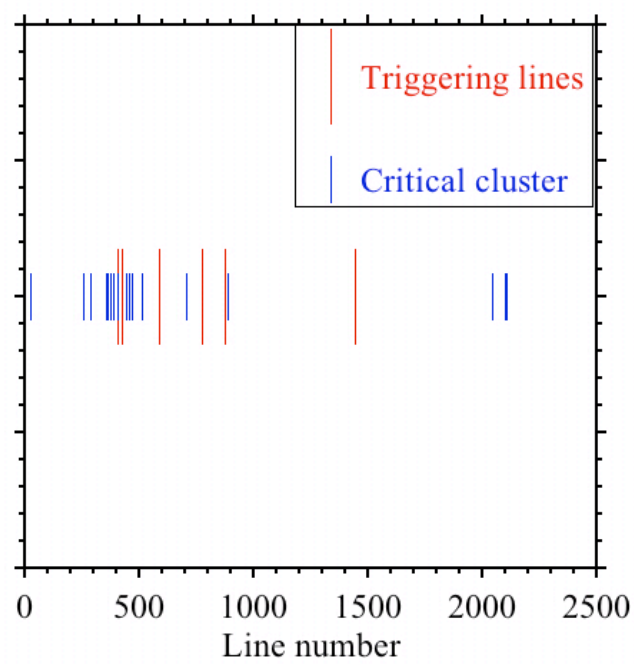

Fig 19. The lines triggering large blackouts and the lines of the dominant critical cluster for the WECC 1553 node network. (AII numbering is artificial to prevent any correspondence with real data).

Fig. 20 shows a zoomed in view of the Fig. 19 for lines numbers between 200 and 600 . From both figures it is clear that there are no lines in common between the two sets.

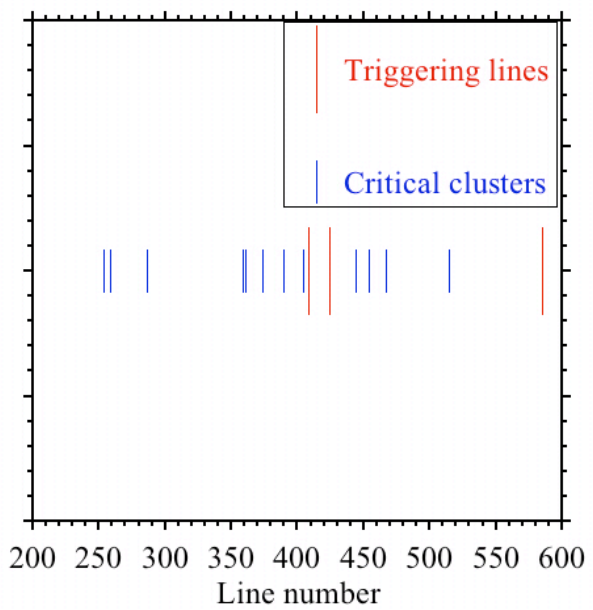

Fig. 20. Zoomed in view of the Fig. 19 for lines numbers between 200 and 600. (All numbering is artificial to prevent any correspondence with real data).

\section{Conclusions}

Cascading failures are made up of both a trigger event and the cascade which itself can vary from one stage to dozens or even hundreds of stages. Therefore, an effective evaluation of the consequences of line outages in a network needs to be done both under widely varying conditions of the network and with many combinations of triggers. The OPA dynamical model allows such an evaluation. Extreme events can be triggered by a few line outages if:

1. The lines are properly selected

2. The network condition or state enables the cascading

In this way, conditions that lead to the extreme events can be identified. This method has been applied here to two WECC network models to identify the most critical lines to trigger such events.

The statistical analysis of the most frequently overloaded lines during numerical simulations of blackouts using the OPA code provides an approach to study the vulnerability of a network model to the propagation of large cascades. It is important to recognize that the already recognized vulnerabilities are the one which will already be protected against, and it is therefore the unknown weaknesses that are likely to cause the rare, large failures.

Using synchronization of the lines during large cascading failures allows us to identify clusters of lines that play a critical role in those cascading failures. Once we have identified the critical clusters, a more detailed analysis of their vulnerability can be carried out. 
The identification of these two types of critical lines, the ones that may trigger an event and the ones that foster its propagation, gives us the information needed in order to apply mitigation strategies to reduce the incidence and consequences of large blackouts.

\section{ACKNOWLEDGMENTS}

We gratefully acknowledge support in part from NSF grants SES-0623985 and SES-0624361 and in part by the California Energy Commission, Public Interest Energy Research Program. This paper does not necessarily represent the views of the Energy Commission, its employees or the State of California. It has not been approved or disapproved by the Energy Commission nor has the Energy Commission passed upon the accuracy or adequacy of the information. One of us (BAC) thanks the financial support of Universidad Carlos III and Banco Santander through a Càtedra de Excelencia.

\section{References}

[1] B.A. Carreras, V.E. Lynch, I. Dobson, D.E. Newman, Complex dynamics of blackouts in power transmission systems, Chaos, vol. 14, no. 3, Sept. 2004, pp. 643-652.

[2] I. Dobson, B.A. Carreras, V.E. Lynch, D.E. Newman, Complex systems analysis of series of blackouts: cascading failure, critical points, and self-organization, Chaos, vol. 17,
026103, June 2007.

[3] H. Ren, I. Dobson, B.A. Carreras, Long-term effect of the $\mathrm{n}-1$ criterion on cascading line outages in an evolving power transmission grid, IEEE Trans. Power Systems, vol. 23, no. 3, August 2008, pp. 1217 - 1225.

[4] R. Gann, J. Venable, E. J. Friedman, A. S. Landsberg, Behavior of coupled automata, Physical Review E, vol. 69, no. 4, 046116, 2004.

[5] B. A. Carreras, D. E. Newman, I. Dobson, and A. B. Poole, Evidence for self organized criticality in a time series of electric power system blackouts, IEEE Transactions on Circuits and Systems Part I, vol. 51, no. 9, pp. 1733-1740, September 2004.

[6] D.E. Newman, B.A. Carreras, V.E. Lynch, I. Dobson, Exploring complex systems aspects of blackout risk and mitigation, IEEE Transactions on Reliability, vol. 60, no. 1, March 2011, pp. 134-143.

[7] Bonneville Power Administration Transmission Services Operations \& Reliability website

http://transmission.bpa.gov/Business/Operations/Outages

[8] I. Dobson, B.A. Carreras, Number and propagation of line outages in cascading events in electric power transmission systems, 48th Annual Allerton Conf. on Communication, Control and Computing, Monticello IL USA, Sept. 2010.

[9] D.E. Newman, B. Nkei, B.A. Carreras, I. Dobson, V.E. Lynch, P. Gradney, Risk assessment in complex interacting infrastructure systems, Thirty-eighth Hawaii International Conference on System Sciences, Hawaii, January 2005. 